\title{
The isoperimetric constant of the random graph process
}

\author{
Itai Benjamini * Simi Haber ${ }^{\dagger}$ Michael Krivelevich $^{\ddagger}$ Eyal Lubetzky ${ }^{\S}$
}

July 6,2018

\begin{abstract}
The isoperimetric constant of a graph $G$ on $n$ vertices, $i(G)$, is the minimum of $\frac{|\partial S|}{|S|}$, taken over all nonempty subsets $S \subset V(G)$ of size at most $n / 2$, where $\partial S$ denotes the set of edges with precisely one end in $S$. A random graph process on $n$ vertices, $\widetilde{G}(t)$, is a sequence of $\left(\begin{array}{l}n \\ 2\end{array}\right)$ graphs, where $\widetilde{G}(0)$ is the edgeless graph on $n$ vertices, and $\widetilde{G}(t)$ is the result of adding an edge to $\widetilde{G}(t-1)$, uniformly distributed over all the missing edges. We show that in almost every graph process $i(\widetilde{G}(t))$ equals the minimal degree of $\widetilde{G}(t)$ as long as the minimal degree is $o(\log n)$. Furthermore, we show that this result is essentially best possible, by demonstrating that along the period in which the minimum degree is typically $\Theta(\log n)$, the ratio between the isoperimetric constant and the minimum degree falls from 1 to $\frac{1}{2}$, its final value.
\end{abstract}

\section{Introduction}

Let $G=(V, E)$ be a graph. For each subset of its vertices, $S \subseteq V$, we define its edge boundary, $\partial S$, as the set of all edges with exactly one endpoint in $S$ :

$$
\partial S=\{(u, v) \in E: u \in S, v \notin S\}
$$

The isoperimetric constant, or isoperimetric number, of $G=(V, E), i(G)$, is defined to be:

$$
i(G)=\min _{\emptyset \neq S \subset V} \frac{|\partial S|}{\min \{|S|,|V \backslash S|\}}=\min _{\substack{\emptyset \neq S \subset V \\|S| \leq \frac{1}{2}|V|}} \frac{|\partial S|}{|S|} .
$$

\footnotetext{
*Weizmann Institute, Rehovot, 76100, Israel. Email: itai.benjamini@weizmann.ac.il

${ }^{\dagger}$ Department of Mathematics, Raymond and Beverly Sackler Faculty of Exact Sciences, Tel Aviv University, Tel Aviv, 69978, Israel. Email: habbersi@tau.ac.il.

${ }^{\ddagger}$ Department of Mathematics, Raymond and Beverly Sackler Faculty of Exact Sciences, Tel Aviv University, Tel Aviv, 69978, Israel. Email: krivelev@post.tau.ac.il. Research supported in part by a USA-Israeli BSF grant and a grant from the Israeli Science Foundation.

${ }^{\S}$ Department of Computer Science, Raymond and Beverly Sackler Faculty of Exact Sciences, Tel Aviv University, Tel Aviv, 69978, Israel. Email: lubetzky@tau.ac.il.
} 
It is well known that this parameter, which measures edge expansion properties of a graph $G$, is strongly related to the spectral properties of $G$, and namely:

$$
\frac{\lambda}{2} \leq i(G) \leq \sqrt{\lambda(2 \Delta(G)-\lambda)}
$$

where $\Delta(G)$ denotes the maximal degree of $G$, and $\lambda$ denotes the second smallest eigenvalue of the Laplacian matrix of $G$ (for proofs of these facts, see [2] and [13]). The upper bound in (11) can be viewed as a discrete version of the Cheeger inequality bounding the first eigenvalue of a Riemannian manifold, and indeed, there is a natural relation between the study of isoperimetric inequalities of graphs and the study of Cheeger constants in spectral geometry. For instance, see [7], where the author relates between isoperimetric constants and spectral properties of graphs and those of certain Riemann surfaces. The eigenvalue bounds in (1) also relate $i(G)$ (as well as a variation of it, the conductance of $G$ ) to the mixing time of a random walk in $G$, defined to be the minimal time it takes a random walk on $G$ to approach the stationary distribution within a variation distance of $1 / 2$.

A closely coupled variant of the isoperimetric constant is the Cheeger constant of a graph, where the edge boundary of $S$ is divided by its volume (defined to be the sum of its degrees) instead of by its size. For further information on this parameter, its relation to the isoperimetric constant, and its corresponding eigenvalue bounds (analogous to (1)), see [8], as well as [9], Chapter 2 .

There has been much study on the isoperimetric constants of various graphs, such as grid graphs, torus graphs, the $n$-cube, and more generally, cartesian products of graphs. See, for instance, [5] 4, 10, 12, 13. In 3], Bollobás studied the isoperimetric constant of random $d$-regular graphs, and used probabilistic arguments to prove that infinitely many $d$-regular graphs $G$ satisfy $i(G) \geq$ $\frac{d}{2}-O(\sqrt{d})$. Alon proved in [1] that this inequality is in fact tight, by providing an upper bound of $i(G) \leq \frac{d}{2}+O(\sqrt{d})$ for any $d$-regular graph $G$ on a sufficiently large number of vertices.

In this paper, we study the isoperimetric constant of general random graphs $\mathcal{G}(n, p), \mathcal{G}(n, M)$, and the random graph process, and show that in these graphs, the ratio between the isoperimetric constant and the minimal degree exhibits an interesting behavior.

We briefly recall several elementary details on these models (for further information, c.f., e.g., 6], Chapter 2). The random graph $\mathcal{G}(n, p)$ is a graph on $n$ vertices, where each pair of distinct vertices is adjacent with probability $p$, and independently of all other pairs of vertices. The distribution of $\mathcal{G}(n, p)$ is closely coupled with that of $\mathcal{G}(n, M)$, a uniform distribution on all graphs on $n$ vertices with precisely $M$ edges, if we choose $p=M /\left(\begin{array}{l}n \\ 2\end{array}\right)$. The random graph process on $n$ vertices, $\widetilde{G}(t)$, is a sequence of $\left(\begin{array}{l}n \\ 2\end{array}\right)$ graphs, where $\widetilde{G}(0)$ is the edgeless graph on $n$ vertices, and $\widetilde{G}(t)$ is the result of adding an edge to $\widetilde{G}(t-1)$, uniformly distributed over all the missing edges. Notice that at a given time $0 \leq t \leq\left(\begin{array}{l}n \\ 2\end{array}\right), \widetilde{G}(t)$ is distributed as $\mathcal{G}(n, M)$ with $M=t$.

For a given graph process $\widetilde{G}$ on $n$ vertices, we define the hitting time of a monotone graph 
property $\mathcal{A}$ (a family of graphs closed under isomorphism and the addition of edges) as:

$$
\tau(\mathcal{A})=\min \left\{0 \leq t \leq\left(\begin{array}{l}
n \\
2
\end{array}\right): \widetilde{G}(t) \in \mathcal{A}\right\}
$$

We use the abbreviation $\tau(\delta=d)$ for the hitting time $\tau(\{G: \delta(G) \geq d\})$ of a given graph process, where $\delta(G)$ denotes the minimal degree of $G$. Finally, we say that a random graph $G$ satisfies some property with high probability, or almost surely, or that almost every graph process satisfies a property, if the probability for the corresponding event tends to 1 as the number of vertices tends to infinity.

Consider the beginning of the random graph process. It is easy to see that for every graph $G, i(G)$ is at most $\delta(G)$, the minimal degree of $G$ (choose a set $S$ consisting of a single vertex of degree $\delta(G))$. Hence, at the beginning of the graph process, $i(\widetilde{G}(0))=0=\delta(\widetilde{G}(0))$, and this remains the case as long as there exists an isolated vertex in $\widetilde{G}(t)$. Next, consider the time where the minimal degree and maximal degree of the random graph process become more or less equal. At this point, we can examine random $\delta$-regular graphs for intuition as to the behavior of the isoperimetric constant, in which case the results of [1] and [3] imply that $i(\widetilde{G}(t))$ is roughly $\delta / 2$. Hence, at some point along the random graph process, the behavior of the isoperimetric constant changes, and instead of being equal to $\delta$ it drifts towards $\delta / 2$ (it is easy to confirm that the isoperimetric constant of the complete graph is $\frac{n-1}{2}$ ). The following results summarize the behavior of the isoperimetric constant of the random graph process (and, resulting from which, of the appropriate random graphs models):

In Section 2 we prove that, for almost every graph process, there is equality between the isoperimetric constant and the minimal degree, as long as the minimal degree is $o(\log n)$. In other words, we prove a hitting time result: the minimal degree increases by 1 exactly when the isoperimetric constant increases by 1 throughout the entire period in which $\delta=o(\log n)$.

Theorem 1.1. Let $\ell=\ell(n)$ denote a function satisfying $\ell(n)=o(\log n)$. Almost every graph process $\widetilde{G}$ on $n$ vertices satisfies $i(\widetilde{G}(t))=\delta(\widetilde{G}(t))$ for every $t \in[0, \tau(\delta=\ell)]$. Furthermore, with high probability, for every such $t$, every set $S$ which attains the minimum of $i(\widetilde{G}(t))$ is an independent set of vertices of degree $\delta(\widetilde{G}(t))$.

In Section 3 we show that the $o(\log n)$ bound in Theorem 1.1 is essentially best possible. Indeed, during the period in which the minimal degree is $\Theta(\log n), i(G)$ drifts towards $\frac{1}{2} \delta(G)$, as the next theorem demonstrates:

Theorem 1.2. For every $0<\varepsilon<\frac{1}{2}$ there exists a constant $C=C(\varepsilon)>0$, such that the random graph $G \sim \mathcal{G}(n, p)$, where $p=C \frac{\log n}{n}$, almost surely satisfies:

$$
i(G) \leq\left(\frac{1}{2}+\varepsilon\right) \delta(G)=\Theta(\log n)
$$

Furthermore, with high probability, every set $S$ of size $\left\lfloor\frac{n}{2}\right\rfloor$ satisfies: $\frac{|\partial S|}{|S|}<\left(\frac{1}{2}+\varepsilon\right) \delta(G)$. 
An analogous statement holds for $\mathcal{G}(n, M)$ as well, where $M=C n \log n$ for a sufficiently large $C=C(\varepsilon)$.

We note that throughout the paper, all logarithms are in the natural basis.

\section{The behavior of $i(G)$ when $\delta=o(\log n)$}

\subsection{Proof of Theorem 1.1}

Since every graph $G$ satisfies $i(G) \leq \delta(G)$, proving that, for every $d \leq \ell$, with high probability, at time $\tau(\delta=d)$ the isoperimetric constant of $G$ is at least $d$, will prove the theorem. We show that

for every $d=d(n)=o(\log n)$, the probability for this event is at least $1-o\left(\frac{1}{\log n}\right)$, and the theorem follows from the union bound on the complement events.

Recall that almost every graph process $\widetilde{G}$ satisfies $\delta(\widetilde{G}) \leq d-1$ at time

$$
m_{d}=\left(\begin{array}{l}
n \\
2
\end{array}\right) \frac{\log n+(d-1) \log \log n-\omega(n)}{n},
$$

and $\delta(\widetilde{G}) \geq d$ at time

$$
M_{d}=\left(\begin{array}{l}
n \\
2
\end{array}\right) \frac{\log n+(d-1) \log \log n+\omega(n)}{n},
$$

where $d \geq 1$ is some fixed integer, the $\omega(n)$-term represents a function growing to infinity arbitrarily slowly while satisfying $\omega(n) \leq \log \log \log n$, and all $\operatorname{logarithms}$ are natural (see, e.g., [6], Chapter $3)$. Hence, $\tau(\delta=d)$ is between $m_{d}$ and $M_{d}$. Using the same methods described in [6], it is easy to extend this statement typically to every $d=d(n)=o(\log n)$, as the next proposition summarizes:

Proposition 2.1. Let $\ell=\ell(n)=o(\log n)$. For every $1 \leq d \leq \ell$ define:

$$
r=r(n)=\frac{\log n}{d}
$$

Next, define the following threshold functions:

$$
m_{d}=\left(\begin{array}{l}
n \\
2
\end{array}\right) \frac{\log n+(d-1) \log r-(2 d+\omega(n))}{n},
$$

and:

$$
M_{d}=\left(\begin{array}{l}
n \\
2
\end{array}\right) \frac{\log n+(d-1) \log r+(2 d+\omega(n))}{n},
$$

where $\omega(n) \leq \log \log r$ and $\lim _{n \rightarrow \infty} \omega(n)=\infty$. Then, almost every graph process $\widetilde{G}$ satisfies $\delta\left(\widetilde{G}\left(m_{d}\right)\right) \leq d-1$ and $\delta\left(\widetilde{G}\left(M_{d}\right)\right) \geq d$ for every $1 \leq d \leq \ell$.

Notice that $r \leq \log n$, and that $r$ tends to infinity as $n \rightarrow \infty$, hence these definitions coincide with the previous definitions of $m_{d}$ and $M_{d}$ for a fixed $d$, and it is left to verify them for $1 \ll d \ll$ $\log n$. Proposition 2.1 follows from standard first moment and second moment considerations, and 
we postpone its proof to Section 2.2. Assume therefore, throughout the proof of Theorem 1.1, that the hitting time $\tau(\delta=d)$ is almost surely in the interval $\left(m_{d}, M_{d}\right]$ for every $1 \leq d \leq \ell$.

Consider a set $S \subset V$ of size $|S| \leq n / 2$; we need to show that, with high probability, every such set satisfies $|\partial S| \geq \delta(\widetilde{G}(t))|S|$ at every time $t \leq \tau(\delta=\ell)$ in the random graph process. Clearly, at a given time $t=M$, the random variable $|\partial S|$ has a binomial distribution with parameters $\mathcal{B}(|S|(n-|S|), p)$, where $p=M /\left(\begin{array}{l}n \\ 2\end{array}\right)$. When $|S|$ is sufficiently large (namely, larger than $n^{1 / 4}$ ), the result follows from standard large deviation bounds and bounds on the tail of the binomial distribution. However, these bounds are not tight enough for small values of $|S|$, which require a separate and more delicate treatment.

Throughout the rest of this section, fix $d=d(n)=o(\log n)$, and define $m_{d}, M_{d}$ and $r$ according to Proposition 2.1

The following lemma shows that every small set $S$ has a boundary of size at least $\delta(G)|S|$ almost surely:

Lemma 2.2. With probability at least $1-o\left(n^{-1 / 5}\right)$, the random graph process $\widetilde{G}$ satisfies that every $G \in\left\{\widetilde{G}(t): m_{d} \leq t \leq \tau(\delta=d)\right\}$ has the property $|\partial S| \geq \delta(G)|S|$ for every set $S$ of size $|S| \leq n^{1 / 4}$. Furthermore, if such a set $S$ satisfies $|\partial S|=\delta(G)|S|$, it is necessarily an independent set of vertices whose degrees are $\delta(G)$.

Proof. Given a graph $G=(V, E)$, we call a set $S \subset V$ bad if it satisfies $|\partial S|<\delta(G)|S|$. The idea of the proof is as follows: we show that, with high probability, every induced subgraph on $k \leq n^{1 / 4}$ vertices has a low average degree. Since bad sets have a boundary of at most $\delta(G)|S|$, this implies that bad sets, as well as sets which are "almost" bad, must contain many vertices whose degrees are low in $G$. The result is derived from several properties of the set of all vertices of low degrees. We begin with defining this set of vertices and examining its properties:

Definition. Let $G=(V, E)$. The set of vertices $\operatorname{Small}(G)$ is defined to be:

$$
\operatorname{SMALL}=\operatorname{SmaLL}(G)=\{v \in V: d(v)<4(d+6)\} .
$$

Claim 2.3. With probability at least $1-o\left(n^{-1 / 5}\right)$, the random graph process $\widetilde{G}$ has the following property: for every $m_{d} \leq t \leq M_{d}$, SMALL is an independent set, and every two vertices of SMALL have no common neighbors in $V$.

Proof. Notice that the set SMALL changes along the random graph process, as vertices are removed from it once they reach a degree of $4(d+6)$. We show a slightly stronger result: if $S_{0}$ denotes $\operatorname{Small}\left(\widetilde{G}\left(m_{d}\right)\right)$, then $S_{0}$ satisfies the above properties almost surely for every $m_{d} \leq t \leq M_{d}$. Since $\operatorname{Small}(\widetilde{G}(t)) \subseteq S_{0}$ for every $t \geq m_{d}$, this will imply the claim. In order to prove this result, we show that, with high probability, $S_{0}$ satisfies the above properties at time $t=m_{d}$, and that the addition of $M_{d}-m_{d}$ edges almost surely does not harm these properties of $S_{0}$. 
Let $p=m_{d} /\left(\begin{array}{l}n \\ 2\end{array}\right)$, and let $G_{0} \sim \mathcal{G}(n, p)$. The same consideration will show that SMALL satisfies the properties of the claim with the mentioned probability, both in $\mathcal{G}(n, p)$ and in $\mathcal{G}\left(n, m_{d}\right)$; for the sake of simplicity, we perform the calculations in the $\mathcal{G}(n, p)$ model, and note that they hold for the $\mathcal{G}\left(n, m_{d}\right)$ model as well. Indeed, the main tool in the proof is an upper bound on the probability for a low degree (a degree of $L=o(n)$ when the edge probability is $p$ ), and the probabilities of the relevant events in $\mathcal{G}\left(n, m_{d}\right)$ are already upper bounded by the corresponding probabilities in $\mathcal{G}(n, p)$.

Both of the properties mentioned in the claim are immediate consequences of the next upper bound for the probability of the event $\{\mathcal{B}(n-L, p) \leq D\}$, where $4 d \leq D \leq 30 d$ and $L=o(n)$. We use the fact that, by this choice of parameters, $D \ll(n-L) p$, implying the following monotonicity of the binomial distribution:

$$
\begin{gathered}
\operatorname{Pr}[\mathcal{B}(n-L, p) \leq D] \leq(D+1)\left(\begin{array}{c}
n-L \\
D
\end{array}\right) p^{D}(1-p)^{n-L-D} \leq \\
\leq(D+1)\left(\frac{\mathrm{e} p n}{D}\right)^{D} \mathrm{e}^{-p(1-o(1)) n} \leq(30 d+1)\left(\frac{(\mathrm{e}+o(1)) \log n}{4 d}\right)^{30 d} \mathrm{e}^{-(1-o(1)) \log n} \leq \\
\leq(30 d+1) r^{30 d} \mathrm{e}^{-(1-o(1)) \log n}=\exp (O(1)+\log d+30 d \log r-(1-o(1)) \log n)= \\
=\exp \left(O(1)+\log d+30 \log n \frac{\log r}{r}-(1-o(1)) \log n\right)=\exp (-(1-o(1)) \log n)=o\left(n^{-0.9}\right) .
\end{gathered}
$$

Set $D=4(d+6)$, and let $A_{u, v}$ denote the event that the edge $(u, v)$ belongs to the induced graph on SMALL, for a given pair of vertices $u, v \in V$. The following holds:

$$
\operatorname{Pr}\left[A_{u, v}\right]=p \operatorname{Pr}[\mathcal{B}(n-2, p)<D-1]^{2} \leq \frac{(1+o(1)) \log n}{n^{2.8}}=o\left(n^{-2.5}\right) .
$$

Thus, the probability that there exists such a pair of vertices is at most $\left(\begin{array}{l}n \\ 2\end{array}\right) \operatorname{Pr}\left[A_{u, v}\right]=o\left(n^{-1 / 2}\right)$, and $\operatorname{Smald}\left(G_{0}\right)$ is an independent set with probability $1-o\left(n^{-1 / 2}\right)$. Next, let $A_{u, v, w}$ denote the event that $u, v \in \operatorname{Smald}\left(G_{0}\right)$ and $w$ is a common neighbor of $u$ and $v$, for some $u, v, w \in V$. Again, we get:

$$
\begin{gathered}
\operatorname{Pr}\left[A_{u, v, w}\right]=p^{2}\left(p \operatorname{Pr}[\mathcal{B}(n-3, p)<D-2]^{2}+(1-p) \operatorname{Pr}[\mathcal{B}(n-3, p)<D-1]^{2}\right) \leq \\
\leq p^{2} n^{-1.8}=o\left(n^{-3.5}\right),
\end{gathered}
$$

and therefore $\left(\begin{array}{l}n \\ 3\end{array}\right) \operatorname{Pr}\left[A_{u, v, w}\right]=o\left(n^{-1 / 2}\right)$.

We have shown that with probability at least $1-o\left(n^{-1 / 2}\right), \operatorname{SmalL}\left(G_{0}\right)$ satisfies the two properties of the claim, and by the same argument, $S_{0}=\operatorname{Small}\left(\widetilde{G}\left(m_{d}\right)\right)$ satisfies the two properties of the claim with probability at least $1-o\left(n^{-1 / 2}\right)$. We now give a rough upper bound on the size of $S_{0}$ using the above upper bound on $\mathcal{B}(n, p)$ :

$$
\mathbb{E}\left|S_{0}\right| \leq n \operatorname{Pr}[\mathcal{B}(n-1, p)<D]=o\left(n^{0.1}\right)
$$


Hence, by Markov's inequality, $\operatorname{Pr}\left[\left|S_{0}\right| \geq n^{0.3}\right] \leq n^{-1 / 5}$. Altogether, we have shown that, with probability $1-o\left(n^{-1 / 5}\right)$, the set SMALL at time $t=m_{d}$ satisfies the requirements of the claim, and is of size at most $n^{0.3}$.

Assume that indeed $\left|S_{0}\right| \leq n^{0.3}$ and that the distance between every pair of vertices of $S_{0}$ is at least 3 at time $m_{d}$. We wish to show that this property is maintained throughout the period $t \in\left(m_{d}, M_{d}\right]$. Notice that the probability that an edge will be added between a given pair of vertices $u, v$ in this period is

$$
\hat{p}=(1+o(1))\left(M_{d}-m_{d}\right) /\left(\begin{array}{l}
n \\
2
\end{array}\right)=(2+o(1)) \frac{2 d+\omega(n)}{n} .
$$

Hence, the probability that an internal edge is added to $S_{0}$ is at most:

$$
\left(\begin{array}{c}
\left|S_{0}\right| \\
2
\end{array}\right) \hat{p} \leq \frac{n^{0.6}(1+o(1))(2 d+\omega(n))}{n}=o\left(n^{-1 / 5}\right) .
$$

Since the set of neighbors of $S_{0}, N\left(S_{0}\right)$, consists of at most $4(d+6)\left|S_{0}\right|$ vertices, the probability that an edge is added between $N\left(S_{0}\right)$ and a vertex of $S_{0}$ is at most:

$$
\left|N\left(S_{0}\right)\right|\left|S_{0}\right| \hat{p} \leq \frac{n^{0.6}(2+o(1)) 4(d+6)(2 d+\omega(n))}{n}=o\left(n^{-1 / 5}\right) .
$$

Finally, the probability that two edges are added between one vertex of $V \backslash S_{0}$ and two vertices of $S_{0}$ is at most:

$$
n\left(\begin{array}{c}
\left|S_{0}\right| \\
2
\end{array}\right) \hat{p}^{2} \leq \frac{n^{1.6}(2+o(1))(2 d+\omega(n))^{2}}{n^{2}}=o\left(n^{-1 / 5}\right) .
$$

Altogether, with probability $1-o\left(n^{-1 / 5}\right)$ the set $S_{0}$ maintains the property that the distance between each pair of its vertices is at least 3 in the period $m_{d} \leq t \leq M_{d}$. This completes the proof of the claim.

The following claim is crucial to the handling of small sets in $G$, showing that the average degree in the induced subgraphs on them is small:

Claim 2.4. With probability at least $1-o\left(n^{-1 / 5}\right)$, the random graph process $\widetilde{G}$ has the following property: for every $t \leq M_{d}$, every induced subgraph of $\widetilde{G}(t)$ on $k \leq n^{1 / 4}$ vertices contains at most $2 k$ edges.

Proof. Since this property is monotone with respect to the removal of edges, it is enough to prove the claim for $t=M_{d}$. Let $p=M_{d} /\left(\begin{array}{l}n \\ 2\end{array}\right)$ and $G \sim \mathcal{G}(n, p)$. Fix $1 \leq k \leq n^{1 / 4}$; the probability that an induced subgraph $H$ on $k$ vertices has at least $2 k$ edges is:

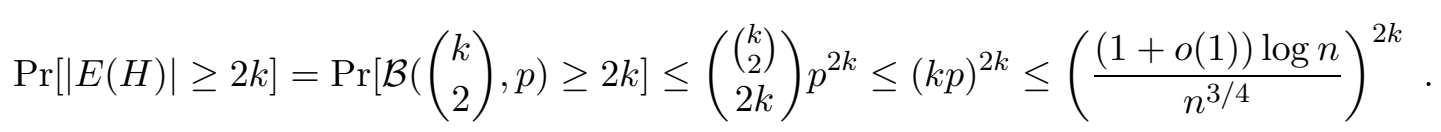


Summing over all the subgraphs of size at most $k$, we obtain that the probability that such a subgraph exists is at most:

$$
\sum_{k \leq n^{1 / 4}} \sum_{|H|=k} \operatorname{Pr}[|E(H)| \geq 2 k] \leq \sum_{k \leq n^{1 / 4}}\left(\begin{array}{l}
n \\
k
\end{array}\right)\left(\frac{(1+o(1) \log n}{n^{3 / 4}}\right)^{2 k} \leq \sum_{k \leq n^{1 / 4}}\left(n^{-\frac{1}{2}+o(1)}\right)^{k}=o\left(n^{-1 / 5}\right) .
$$

Again, performing the same calculation in $\mathcal{G}\left(n, M_{d}\right)$ gives the same result: the probability that a specific set of $2 k$ edges belongs to $\mathcal{G}\left(n, M_{d}\right)$ is $\left(\begin{array}{c}N-2 k \\ M_{d}-2 k\end{array}\right) /\left(\begin{array}{c}N \\ M_{d}\end{array}\right)$ (where $N=\left(\begin{array}{l}n \\ 2\end{array}\right)$ ), which equals $\left((1+o(1)) M_{d} / N\right)^{2 k}=((1+o(1)) p)^{2 k}$.

Equipped with Claim 2.3 and Claim 2.4, we are ready to prove Lemma 2.2.

Recall that a set $S$ is bad iff $|\partial S|<\delta(G)|S|$. We call a bad set $S$ elementary if it does not contain a smaller bad set, i.e., every $T \subset S, T \neq S$ is not bad. Clearly, in order to show that there are no bad sets of size at most $n^{1 / 4}$, it is enough to show that there are no elementary bad sets of such size. With high probability, every $G \in\left\{\widetilde{G}(t): m_{d} \leq t \leq M_{d}\right\}$ satisfies both Claim [2.3 and Claim 2.4. Since $m_{d}<\tau(\delta=d) \leq M_{d}$, every graph $G=\widetilde{G}(t)$ in the interval $m_{d} \leq t \leq \tau(\delta=d)$ satisfies both claims, as well as $\delta(G) \leq d$. We claim that this implies the required result; to see this, consider a graph $G$ which satisfies the above properties, and let $\delta=\delta(G)$. We first prove that there are no elementary bad sets of size at most $n^{1 / 4}$ in $G$ :

Let $S$ denote an elementary bad set $S$ of size $k \leq n^{1 / 4}$. Notice that necessarily $k \geq 2$, since a single vertex has at least $\delta$ edges and hence cannot account for a bad set. By Claim 2.4 the induced graph $H$ on $S$ contains at most $2 k$ edges. Since the boundary of $S$ contains at most $\delta k-1 \leq d k$ edges, this implies that $\mid S \cap$ SMALL $\mid \geq \frac{3}{4} k$, otherwise the number of edges in $H$ would satisfy:

$$
|E(H)|=\frac{1}{2} \sum_{v \in S} d_{H}(v) \geq \frac{1}{2}\left(\frac{k}{4} 4(d+6)-d k\right) \geq 3 k,
$$

leading to a contradiction. Assume therefore that at most $k / 4$ vertices in $S$ do not belong to SmaLL. We define $A=S \cap$ Small, and $B=S \backslash A$. By Claim [2.3. $A$ is an independent set, and furthermore, no two vertices of $A$ have a common neighbor in $B$. Hence, each vertex of $B$ is adjacent to at most one vertex of $A$, and if we denote by $A^{\prime} \subseteq A$ the vertices of $A$, which are not adjacent to any vertex of $S$, the following holds:

$$
\left|A^{\prime}\right| \geq|A|-|B| \geq\left(\frac{3}{4}-\frac{1}{4}\right) k=\frac{1}{2} k
$$

In particular, $A^{\prime}$ is nonempty; we claim that this contradicts the fact that $S$ is elementary. Indeed, each vertex $v \in A^{\prime}$ is not adjacent to any vertex in $S$, hence it contributes $d(v)$ edges to $\partial S$. Removing the vertex $v$ would result in a nonempty $(k \geq 2)$ strictly smaller subset $T$ of $S$ which satisfies:

$$
|\partial T|=|\partial S|-d(v) \leq|\partial S|-\delta<\delta(|S|-1)=\delta|T|,
$$

establishing a contradiction. We conclude that $G$ does not contain bad sets of size at most $n^{1 / 4}$.

Next, consider a set $S$ of size $|S| \leq n^{1 / 4}$ which satisfies $|\partial S|=\delta|S|$. If $|S|=1$, obviously $S$ consists of a single vertex of degree $\delta$ and we are done. Otherwise, repeating the above arguments 
for bad sets, we deduce that $|S \cap \operatorname{SMALL}| \geq \frac{3}{4}|S|$ (this argument merely required that $|\partial S| \leq \delta|S|$ ) and that $S$ contains a nonempty set $A^{\prime}$, whose vertices are not adjacent to any vertex of $S$. Consider a vertex $v \in A^{\prime}$; this vertex contributes $d(v) \geq \delta$ edges to $\partial S$. However, $d(v)$ cannot be greater than $\delta$, otherwise the set $S^{\prime}=S \backslash\{v\}$ would satisfy $\left|\partial S^{\prime}\right|<\delta\left|S^{\prime}\right|$, contradicting the fact that there are no bad sets of size at most $n^{1 / 4}$ in $G$. Therefore, all the vertices of $A^{\prime}$ are of degree $\delta$, and are not adjacent to any of the vertices of $S$. If we denote the remaining vertices by $S^{\prime}=S \backslash A^{\prime}, S^{\prime}$ satisfies $\left|\partial S^{\prime}\right|=\delta|S|-\delta\left|A^{\prime}\right|=\delta\left|S^{\prime}\right|$, and, by induction, the result follows.

This completes the proof of Lemma 2.2 .

The large sets are handled by the following lemma, which shows that even at time $m_{d}$ (when the minimal degree is still at most $d-1$ ) these sets already have boundaries of size at least $d|S|+1$.

Lemma 2.5. With probability at least $1-o\left(n^{-1 / 5}\right)$, the graph $\widetilde{G}\left(m_{d}\right)$ satisfies $|\partial S|>d|S|$ for every set $S$ of size $n^{1 / 4} \leq|S| \leq n / 2$ (and hence $\widetilde{G}(t)$ has this property for every $t \geq m_{d}$ with probability at least $\left.1-o\left(n^{-1 / 5}\right)\right)$.

Proof. Define $p=m_{d} /\left(\begin{array}{l}n \\ 2\end{array}\right)$. For the sake of simplicity, the calculations are performed in the $\mathcal{G}(n, p)$ model and we note that by the same considerations the results apply for the corresponding $\mathcal{G}\left(n, m_{d}\right)$ model as well. To show that, with probability $1-o\left(n^{-1 / 5}\right)$, the random graph $G \sim \mathcal{G}(n, p)$ satisfies $|\partial S|>d|S|$ for sets $S$ of the given size, argue as follows:

Fix a set $S \subset V$ of size $k, \frac{n}{\log n} \leq k \leq n / 2$, and let $A_{S}$ denote the event $\{|\partial S| \leq d k\}$. Let $\mu$ denote $\mathbb{E}|\partial S|=k(n-k) p$. By the Chernoff bound, $\operatorname{Pr}[|\partial S|<\mu-t] \leq \exp \left(-\frac{1}{2 \mu} t^{2}\right)$. Therefore, setting $t=\mu-(d k+1)$, we get:

$$
\begin{gathered}
\operatorname{Pr}\left[A_{S}\right]=\operatorname{Pr}[|\partial S|<d k+1] \leq \exp \left(-\frac{1}{2}\left(1-\frac{d+\frac{1}{k}}{(n-k) p}\right)^{2} k(n-k) p\right) \leq \\
\leq \exp \left(-\frac{1}{2}\left(1-\frac{(2+o(1)) d}{\log n}\right)^{2} k\left(\frac{1}{2}-o(1)\right) \log n\right)=\exp \left(-\frac{1-o(1)}{4} k \log n\right) .
\end{gathered}
$$

Hence, the probability that there exists such a set $S$ is at most:

$$
\begin{aligned}
& \sum_{k=\frac{n}{\log n}}^{n / 2}\left(\begin{array}{l}
n \\
k
\end{array}\right) \exp \left(-\frac{1-o(1)}{4} k \log n\right) \leq \sum_{k=\frac{n}{\log n}}^{n / 2}\left(\mathrm{e} \frac{n}{k}\right)^{k} \exp \left(-\frac{1-o(1)}{4} k \log n\right) \leq \\
\leq & \sum_{k=\frac{n}{\log n}}^{n / 2} \exp \left(k(\log \log n+1)-\frac{1-o(1)}{4} k \log n\right) \leq \sum_{k=\frac{n}{\log n}}^{n / 2}\left(n^{-\frac{1}{4}+o(1)}\right)^{k}=o\left(n^{-1 / 5}\right) .
\end{aligned}
$$

Let $S \subset V$ be a set of size $n^{1 / 4} \leq k \leq \frac{n}{\log n}$. Notice that:

$$
(n-k) p=(1+o(1)) \log n
$$


and hence, $d k<\mu$, and we can give the following upper bound on the probability that $|\partial S| \leq d k$ :

$$
\begin{aligned}
& \operatorname{Pr}[|\partial S| \leq d k] \leq(d k+1) \operatorname{Pr}[|\partial S|=d k]=(d k+1)\left(\begin{array}{c}
k(n-k) \\
d k
\end{array}\right) p^{d k}(1-p)^{k(n-k)-d k} \leq \\
\leq & (d k+1)\left(\frac{\mathrm{e} k(n-k) p}{d k}\right)^{d k} \mathrm{e}^{-p k(n-k-d)}=(d k+1)(\mathrm{e} / d)^{d k}(p(n-k))^{d k} \mathrm{e}^{-k p n+p k^{2}+p k d} .
\end{aligned}
$$

We now use (4) and the facts that $p k \leq 1+o(1)$ and $d=o(k)$, and obtain:

$$
\operatorname{Pr}[|\partial S| \leq d k] \leq O(1) d k(\mathrm{e} / d)^{d k} \frac{(\log n)^{d k} \mathrm{e}^{(2 d+\omega(n)+1+o(1)) k+d}}{n^{k} r^{k(d-1)}} \leq\left(\frac{\mathrm{e}^{\omega(n)+2 d+O(1)} \log n}{n}\right)^{k} .
$$

Summing over all sets $S$ of size $k$, we get:

$$
\begin{aligned}
\sum_{|S|=k} \operatorname{Pr}[|\partial S| \leq d k] & \leq\left(\frac{\mathrm{e} n}{k}\right)^{k}\left(\frac{\mathrm{e}^{\omega(n)+2 d+O(1)} \log n}{n}\right)^{k}=\left(\frac{\mathrm{e}^{\omega(n)+2 d+O(1)} \log n}{k}\right)^{k} \leq \\
& \leq\left(\frac{O(1) n^{2 / r} \log r \log n}{n^{1 / 4}}\right)^{k}=\left(n^{-\frac{1}{4}+o(1)}\right)^{k} .
\end{aligned}
$$

Thus:

$$
\sum_{n^{1 / 4} \leq|S| \leq \frac{n}{\log n}} \operatorname{Pr}[|\partial S| \leq d|S|] \leq \sum_{k \geq n^{1 / 4}}\left(n^{-\frac{1}{4}+o(1)}\right)^{k}=o\left(n^{-1 / 5}\right)
$$

Since $\widetilde{G}$ satisfies the properties of both Lemma 2.2 and Lemma 2.5 for a given $d \leq \ell=o(\log n)$ with probability at least $1-o\left(n^{-1 / 5}\right)$, the union bound over all possible values of $d$ implies that these properties are satisfied almost surely for every $d \leq \ell$. Theorem 1.1 follows directly: to see this, assume that indeed a random graph process $\widetilde{G}$ satisfies the mentioned properties for every $d \leq \ell$, and consider some $d \leq \ell$. By the properties of Lemma 2.2 in the period $t \in\left[m_{d}, \tau(\delta=d)\right]$ every set of size $k \leq n^{1 / 4}$ has at least $\delta k$ edges in its corresponding cut, and if there are precisely $\delta k$ edges in the cut, then $S$ is an independent set of vertices of degree $\delta$. In particular, at time $t=\tau(\delta=d)$, every set $S$ of at most $n^{1 / 4}$ vertices has a ratio $\frac{|\partial S|}{|S|}$ of at least $d$, and a ratio of precisely $d$ implies that $S$ is an independent set of vertices of degree $d$. By monotonicity, this is true for every $t \in[\tau(\delta=d), \tau(\delta=d+1))$. Next, by the properties of Lemma 2.5] every set of size $k \geq n^{1 / 4}$ has at least $d k+1$ edges in its corresponding cut at time $t=m_{d}$. In particular, for every $t \in[\tau(\delta=d), \tau(\delta=d+1))$, every set $S$, larger than $n^{1 / 4}$ vertices, has a ratio $\frac{|\partial S|}{|S|}$ strictly larger than $d$. These two facts imply the theorem.

\subsection{Proof of Proposition 2.1}

A standard first moment consideration shows that indeed, with high probability, $\delta\left(\mathcal{G}\left(n, M_{d}\right)\right) \geq d$ for every $d \leq \ell$. We perform the calculations in the $\mathcal{G}(n, p)$ model and note that the same applies to $\mathcal{G}\left(n, M_{d}\right)$. 
For each $v \in V(G)$, let $A_{v}$ and $B_{v}$ denote the events $\{d(v)=d-1\}$ and $\{d(v) \leq d-1\}$ respectively, and set $Y_{d}=|\{v: d(v)=d-1\}|$ and $Z_{d}=|\{v: d(v) \leq d-1\}|$. Recall that $d=o(\log n)$, and furthermore, we may assume that $d$ tends to infinity as $n \rightarrow \infty$, since $m_{d}$ and $M_{d}$ coincide with the well known threshold functions for constant values of $d$. Choosing $p=M_{d} /\left(\begin{array}{c}n \\ 2\end{array}\right)$, the following holds:

$$
\begin{gathered}
\operatorname{Pr}\left[A_{v}\right]=\left(\begin{array}{c}
n-1 \\
d-1
\end{array}\right) p^{d-1}(1-p)^{n-d} \leq\left(\frac{(1+o(1)) \mathrm{e} \log n}{d}\right)^{d-1} \mathrm{e}^{-\left(1-\frac{d}{n}\right)(\log n+(d-1) \log r+2 d+\omega(n))} \leq \\
\leq \frac{1}{n^{1-d / n}}\left(\frac{(1+o(1)) \mathrm{e} r}{r^{1-d / n}}\right)^{d-1} \mathrm{e}^{-(1-o(1))(2 d+\omega(n))}= \\
=\frac{n^{d / n}}{n}\left((1+o(1)) \mathrm{e} r^{\frac{\log n}{r n}}\right)^{d-1} \mathrm{e}^{-(1-o(1))(2 d+\omega(n))} \leq \frac{1}{n} \mathrm{e}^{-(1-o(1))(d+\omega(n))} .
\end{gathered}
$$

Since $d \leq(n-1) p$, we have:

$$
\operatorname{Pr}\left[B_{v}\right] \leq d \operatorname{Pr}\left[A_{v}\right] \leq \frac{1}{n} \mathrm{e}^{-(1-o(1))(d+\omega(n))} .
$$

Hence,

$$
\mathbb{E} Z_{d} \leq \mathrm{e}^{-(1-o(1))(d+\omega(n))},
$$

and summing over every $d \leq \ell$ we obtain:

$$
\sum_{1 \ll d \leq \ell} \operatorname{Pr}\left[Z_{d}>0\right] \leq \mathrm{e}^{-(1-o(1)) \omega(n)} \sum_{1 \ll d \leq \ell} \mathrm{e}^{-(1-o(1)) d}=o(1)
$$

A second moment argument proves that almost surely $\delta(\mathcal{G}(n, p)) \leq d-1$ for every $d \leq \ell$. To see this, argue as follows (again, calculations are performed in the $\mathcal{G}(n, p)$ model): following the same definitions, only this time with $p=m_{d} /\left(\begin{array}{l}n \\ 2\end{array}\right)$, apply the bound $\left(\begin{array}{l}a \\ b\end{array}\right) \geq\left(\frac{a}{b}\right)^{b}$ and the well known bound $1-x \geq \mathrm{e}^{-x /(1-x)}$ for $0 \leq x<1$, to obtain:

$$
\begin{gathered}
\operatorname{Pr}\left[A_{v}\right]=\left(\begin{array}{c}
n-1 \\
d-1
\end{array}\right) p^{d-1}(1-p)^{n-d} \geq \\
\geq\left(\frac{(1+o(1)) \log n}{d}\right)^{d-1} \mathrm{e}^{(-\log n-(d-1) \log r+2 d+\omega(n)) /(1-p)} \geq \frac{1}{n} \Omega\left(\mathrm{e}^{d+\omega(n)}\right),
\end{gathered}
$$

where in the last inequality we omitted the the $1 /(1-p)$ factor in the exponent, since, for instance, $n^{1-\frac{1}{1-p}}=n^{\frac{-p}{1-p}} \geq n^{-O(1) \frac{\log n}{n}}=\mathrm{e}^{o(1)}$. Therefore:

$$
\mathbb{E} Y_{d}=\Omega\left(\mathrm{e}^{d+\omega(n)}\right)
$$

Take $u \neq v \in V(G)$; denoting $\mathcal{P}_{L}^{K}=\operatorname{Pr}[\mathcal{B}(K, p)=L]$, the following holds:

$$
\operatorname{Cov}\left(A_{u}, A_{v}\right)=\operatorname{Pr}\left[A_{u} \wedge A_{v}\right]-\operatorname{Pr}\left[A_{u}\right] \operatorname{Pr}\left[A_{v}\right]=p\left(\mathcal{P}_{d-2}^{n-2}\right)^{2}+(1-p)\left(\mathcal{P}_{d-1}^{n-2}\right)^{2}-\left(\mathcal{P}_{d-1}^{n-1}\right)^{2}
$$


Since $\mathcal{P}_{d-1}^{n-1}=p \mathcal{P}_{d-2}^{n-2}+(1-p) \mathcal{P}_{d-1}^{n-2}$, we get:

$$
\begin{aligned}
\operatorname{Cov}\left(A_{u}, A_{v}\right) & =p(1-p)\left(\mathcal{P}_{d-2}^{n-2}\right)^{2}+(1-p) p\left(\mathcal{P}_{d-1}^{n-2}\right)^{2}-2 p(1-p) \mathcal{P}_{d-2}^{n-2} \mathcal{P}_{d-2}^{n-2}= \\
& =p(1-p)\left(\mathcal{P}_{d-1}^{n-2}-\mathcal{P}_{d-2}^{n-2}\right)^{2} \leq p\left(\mathcal{P}_{d-1}^{n-2}\right)^{2}
\end{aligned}
$$

Notice that $\mathcal{P}_{d-1}^{n-2}$ corresponds to the event $A_{v}$ for a graph on $n-1$ vertices, and hence a similar calculation to the one in (51) shows that $\mathcal{P}_{d-1}^{n-2}=O(\exp (3 d+\omega(n)) / n)$. Altogether we get:

$$
\operatorname{Cov}\left(A_{u}, A_{v}\right) \leq O(1) p \frac{\mathrm{e}^{6 d+2 \omega(n)}}{n^{2}} \leq O(1) \mathbb{E} Y_{d} \frac{\mathrm{e}^{5 d+\omega(n)} \log n}{n^{3}}=o\left(n^{-2}\right) \mathbb{E} Y_{d},
$$

which gives the following upper bound on the variance of $Y_{d}$ :

$$
\operatorname{Var}\left(Y_{d}\right) \leq \mathbb{E} Y_{d}+\sum_{u \neq v} \operatorname{Cov}\left(A_{u}, A_{v}\right) \leq \mathbb{E} Y_{d}+n^{2} o\left(n^{-2}\right) \mathbb{E} Y_{d}=_{d}(1+o(1)) \mathbb{E} Y_{d}
$$

Applying Chebyshev's inequality gives:

$$
\operatorname{Pr}\left[Y_{d}=0\right] \leq \frac{\operatorname{Var}\left(Y_{d}\right)}{\left(\mathbb{E} Y_{d}\right)^{2}} \leq \frac{1+o(1)}{\mathbb{E} Y_{d}} \leq O\left(\mathrm{e}^{-d-\omega(n)}\right)
$$

and summing over every $d \leq \ell$ we obtain:

$$
\sum_{1 \ll d \leq \ell} \operatorname{Pr}\left[Y_{d}=0\right] \leq O(1) \mathrm{e}^{-\omega(n)} \sum_{1 \ll d \leq \ell} \mathrm{e}^{-d}=o(1)
$$

as required.

\section{The behavior of $i(G)$ when $\delta=\Omega(\log n)$}

Proof of Theorem 1.2 A bisection of a graph $G$ on $n$ vertices is a partition of the vertices into two disjoint sets $(S, T)$, where $|S|=\left\lfloor\frac{n}{2}\right\rfloor$ and $T=V \backslash S$. Fix $\varepsilon_{1}>0$; we first prove that, with high probability, every bisection $(S, V \backslash S)$ of $\mathcal{G}(n, p)$ has strictly less than $\left(\frac{1}{2}+\varepsilon_{1}\right) n p|S|$ edges in the cut it defines, provided that $\lim _{n \rightarrow \infty} n p=\infty$. We omit the floor and ceiling signs to simplify the presentation of the proof.

Let $S$ be an arbitrary set of $n / 2$ vertices. The number of edges in the boundary of $S$ has a binomial distribution with parameters $\mathcal{B}\left(n^{2} / 4, p\right)$, hence (by our assumption on $p$ ) its expected value $\mu$ tends to infinity faster than $n$. By the Chernoff bound, $\operatorname{Pr}[|\partial S| \geq(1+t) \mu] \leq \exp \left(-\mu t^{2} / 4\right)$ provided that $t<2 \mathrm{e}-1$, thus we get:

$$
\operatorname{Pr}\left[|\partial S| \geq\left(\frac{1}{2}+\varepsilon_{1}\right) n p|S|\right]=\operatorname{Pr}\left[|\partial S| \geq\left(1+2 \varepsilon_{1}\right) \mu\right] \leq \exp (-\Omega(\mu)) .
$$

Since this probability is $o\left(2^{-n}\right)$, the expected number of bisections, in which the corresponding cuts contain at least $\left(\frac{1}{2}+\varepsilon_{1}\right) n p|S|$ edges, is $o(1)$. 
Next, fix $\varepsilon_{2}>0$. We claim that the minimal degree of $\mathcal{G}(n, p)$, where $p=C \frac{\log n}{n}$ and $C=C\left(\varepsilon_{2}\right)$ is sufficiently large, is at least $\left(1-\varepsilon_{2}\right) n p$. Applying the Chernoff bound on the binomial distribution representing the degree of a given vertex $v$ gives:

$$
\operatorname{Pr}\left[d(v) \leq\left(1-\varepsilon_{2}\right) n p\right]=\operatorname{Pr}\left[d(v) \leq\left(1-\varepsilon_{2}+o(1)\right) \mathbb{E} d(v)\right] \leq \exp \left(-C \frac{\varepsilon_{2}^{2}}{2}(1-o(1)) \log n\right),
$$

and for $C>\frac{2}{\varepsilon_{2}^{2}}$ this probability is smaller than $\frac{1}{n}$.

Altogether, for a sufficiently large $C$, the following holds with high probability: every bisection $(S, V \backslash S)$ satisfies:

$$
\frac{|\partial S|}{|S|}<\frac{\frac{1}{2}+\varepsilon_{1}}{1-\varepsilon_{2}} \delta(G)=\left(\frac{1}{2}+\varepsilon\right) \delta(G)
$$

where $\varepsilon=\frac{\varepsilon_{1}+\varepsilon_{2} / 2}{1-\varepsilon_{2}}$.

Remark 3.1: We note that the above argument gives a crude estimate on the value of $C=C(\varepsilon)$. Since the first claim, concerning the behavior of bisections, holds for every value of $C$, we are left with determining when typically the minimal degree of $G$ becomes sufficiently close to the average degree. This threshold can be easily computed, following arguments similar to the ones in the proof of Proposition 2.1 the following value of $C(\varepsilon)$ is sufficient for the properties of the theorem to hold with high probability:

$$
C>\frac{1+2 \varepsilon}{2 \varepsilon-\log (1+2 \varepsilon)} .
$$

Remark 3.2: Theorem 1.2 provides an upper bound on $i(G)$, which is almost surely arbitrarily close to $\frac{\delta}{2}$ while the graph satisfies $\delta=\Theta(\log n)$. We note that the arguments of Theorem 1.1 can be repeated (in a simpler manner) to show that with high probability $i(\widetilde{G}(t)) \geq \delta / 2$ for every $t$, and hence the bound in Theorem 1.2 is tight.

\section{Concluding remarks}

We have shown that there is a phase transition when the minimal degree changes from $o(\log n)$ to $\Omega(\log n)$; it would be interesting to give a more accurate description of this phase transition. Theorem 1.1 treats $\delta(G)=o(\log n)$, and Theorem 1.2 shows that, almost surely, $i(G)<\delta(G)$ once $p=C \log n / n$, where $X>2 /(1-\log 2) \approx 6.52$, in which case $\delta(G)>(C / 2) \log n$. Hence we are left with the period in which $\delta(G)=c \log n$, where $0<c \leq 1 /(1-\log 2) \approx 3.26$. It seems plausible to show that in this period $i(G)=\delta(G)$, i.e., that the isoperimetric constant is determined either by the typical minimal degree, or by the typical size of a bisection.

The vertex version of the isoperimetric constant (minimizing the ratio $|\delta S| /|S|$, where $\delta S \subset V \backslash S$ is the vertex neighborhood of $S$ ) is less natural, since the minimum has to be defined on all nonempty sets of size at most $n /(K+\varepsilon)$ if we wish to allow the constant to reach the value $K$. Nevertheless, 
the methods used to prove Theorem 1.1 can prove similar results for the vertex case, at least as long as the minimum degree is constant. Indeed, in that case, the probability for two vertices to have a common neighbor is small enough not to have an effect on the results.

Finally, it is interesting to consider the isoperimetric constant of certain subgraphs along the random graph process. To demonstrate this, we consider the period of $\widetilde{G}$ in which the minimal degree is 0 , i.e., $t \leq \tau(\delta=1)$. The existence of isolated vertices in $\widetilde{G}(t)$ implies that $i(\widetilde{G}(t))=0$, however even if we disregard these vertices, and examine $G^{\prime}(t)$, the induced subgraph on the nonisolated vertices, then after a short while (say, at $t=c n$ for some $c>0$ ), $i\left(G^{\prime}(t)\right)<\varepsilon$ for every $\varepsilon>0$. An easy calculation shows that small sets, with high probability, have an edge boundary which is smaller than their size. For instance, when $p=c / n$ for some $c<1, \mathcal{G}(n, p)$ almost surely satisfies that all connected components are of size $O(\log n)$, hence each component $\mathcal{C}$ has a ratio $\frac{|\partial \mathcal{C}|}{|\mathcal{C}|}$ of 0 . Furthermore, if we take $p=C / n$ for some $C>1$, and consider the giant component $H$ (recall that for this value of $p$, almost surely there is a single component of size $\Theta(n)$, and all other components are of size $O(\log n)), i(H)<\varepsilon$ for every $\varepsilon>0$. One way to see this, is to consider a collection of arbitrarily long paths, each of which connects to the giant component at precisely one end.

Acknowledgement The authors would like to thank Noga Alon for helpful discussions and keen observations.

\section{References}

[1] N. Alon, On the edge-expansion of graphs, Combinatorics, Probability and Computing 6 (1997), 145-152.

[2] N. Alon and V.D. Milman, $\lambda_{1}$, isoperimetric inequalities for graphs and superconcentrators, J. Combinatorial Theory, Ser. B 38 (1985), 73-88.

[3] B. Bollobás, The isoperimetric number of random regular graphs, Europ. J. Combinatorics 9 (1988), 241-244.

[4] B. Bollobás and I. Leader, An isoperimetric inequality on the discrete torus, SIAM J. Disc. Math. 3 (1990) 32-37.

[5] B. Bollobás and I. Leader, Edge-isoperimetric inequalities in the grid, Combinatorica 11(1991) 299-314.

[6] B. Bollobás, Random graphs, volume 73 of Cambridge Studies in Advanced Mathematics, Cambridge University Press, Cambridge, second edition, 2001.

[7] P. Buser, Cubic graphs and the first eigenvalue of a Riemann surface, Mathematische Zeitschrift, 162 (1978), 87-99. 
[8] F.R.K. Chung, Laplacians of graphs and Cheegers inequalities, in: Proc. Int. Conf. "Combinatorics, Paul Erdős is Eighty", Keszthely (Hungary), 1993, 2, 116.

[9] F.R.K. Chung, Spectral Graph Theory, American Mathematical Society, no. 92 in the Regional Conference Series in Mathematics, Providence, RI, 1997.

[10] F.R.K. Chung and P. Tetali, Isoperimetric inequalities for cartesian products of graphs, Combinatorics, Probability and Computing 7 (1998), 141-148.

[11] J. Friedman, J. Kahn and E. Szemerédi, On the second eigenvalue in random regular graphs, Proc. 21st ACM STOC (1989), 587-598.

[12] C. Houdré and P. Tetali, Isoperimetric constants for product Markov chains and graph products, Combinatorica Vol. 24 (2004), 359-388.

[13] B. Mohar, Isoperimetric numbers of graphs, Journal of Combinatorial Theory, Series B, 47 (1989), 274-291.

[14] E. Shamir and E. Upfal, On factors in random graphs, Israel J. Math. 39 (1981), no. 4, 296-302. 